\title{
Towards Describing Multi-fractality of Traffic Using Local Hurst Function
}

\author{
Ming $\mathrm{Li}^{1}$, S.C. $\mathrm{Lim}^{2}$, Bai-Jiong $\mathrm{Hu}^{1}$, and Huamin $\mathrm{Feng}^{3}$ \\ ${ }^{1}$ School of Information Science \& Technology, East China Normal University, \\ Shanghai 200062, P.R. China \\ ming_lihk@yahoo.com, hubjune@gmail.com \\ ${ }^{2}$ Faculty of Engineering, Multimedia University, 63100 Cyberjaya, Selanger, Malaysia \\ sclim@mmu.edu.my \\ ${ }^{3}$ Key Laboratory of Security and Secrecy of Information, Beijing Electronic Science and \\ Technology Institute, Beijing 100070, P.R. China \\ fenghmabesti.edu.cn
}

\begin{abstract}
Long-range dependence and self-similarity are two basic properties of network traffic time series. Fractional Brownian motion ( $\mathrm{fBm}$ ) and its increment process fractional Gaussian noise (fGn) are commonly used to model network traffic with the Hurst index $H$ that determines both the regularity of the sample paths and the long memory property of traffic. However, it appears too restrictive for traffic modeling since it can only model sample paths with the same smoothness for all time parameterized by a constant $H$. A natural extension of $\mathrm{fBm}$ is multifractional Brownian motion $(\mathrm{mBm})$, which is indexed by a time-dependent Hurst index $H(t)$. The main objective of this paper is to model multi-fractality of traffic using $H(t)$, i.e., $\mathrm{mBm}$, on a point-by-point basis instead of an interval-by-interval basis as traditionally done in computer networks. The numerical results for $H(t)$ of real traffic, which are demonstrated in this paper, show that $H(t)$ of traffic is time-dependent, which not only provide an alternative evidence of the multifractal phenomena of traffic but also reveal an challenging issue in traffic modeling: multi-fractality modeling of traffic.
\end{abstract}

Keywords: Network traffic modeling, fractals, multi-fractals, multifractional Brownian motion, local Hurst function.

\section{Introduction}

Experimental observations of long-range dependence (LRD) and self-similarity (SS) of traffic time series in computer communication networks (traffic for short) were actually noted before the eighties of last century [1]. The description of traffic in [1] was further studied and called "packet trains" during the eighties of last century [2]. However, the properties of LRD and SS of traffic were not investigated from a view of self-affine random functions, such as fractional Brownian motion $(\mathrm{fBm})$ or fractional Gaussian noise (fGn), until the last decade, see e.g. [3], [4], [5], [6], [7], and our previous work [8], [9], [10], [11], [12]. 
Further research of traffic exhibits that traffic has multifractal behavior at small time-scales. However, the multifractal behavior of traffic is conventionally described on an interval-by-interval basis by using $H(n)$, where $H(n)$ is the Hurst parameter in the $n$th interval for $n=1,2, \cdots$, see e.g. [13], [14], [15], [16], [17], [18], and our recent work [19]. Note that $H$ plays a role in computer networks, see e.g. [20], [21], and our recent papers [19], [22], [23]. Hence, modeling multi-fractality of traffic becomes a contemporary topic in traffic modeling. From a practice view, any models of multi-fractal phenomena of traffic are desired as they may be promising to understand and or find solutions to some difficult issues, such as simulation of the Internet, performance evaluation, network security, and so forth, in networking as can be seen from [15], [19], [34]. [35].

Owing to the fact that a monofractal model utilizes fractional Brownian motion (fBm) with the constant Hurst index $H$ that characterizes the global self-similarity, see e.g. [24], we need studying the possible variation of scaling behavior locally. To do so, $\mathrm{fBm}$ can be generalized to multifractional Brownian motion $(\mathrm{mBm})$ by replacing the constant $H$ with a time-dependent Hurst function $H(t)$, which is also called the local Holder exponent see e.g. [26], [27], and our work [28], [29], [30]. In this paper, we discuss and describe the multi-scale and multi-fractal properties of real traffic based on $H(t)$. We shall exhibit that $H(t)$ of traffic change erratically with location $t$. It is noted that if $H(t)$ is allowed to be a random function or a random process, then the $\mathrm{mBm}$ is a multifractal process. We note that $H(t)$ differs substantially from $H(n)$ on an interval-by-interval since it can reflect the multifractal behaviors on a point-by-point basis. To the best of our knowledge, modeling multi-scaled and multi-fractal phenomena of real traffic using $H(t)$ is rarely seen.

The rest of paper is organized as follows. We address modelling the multi-fractality of traffic based on the local Hurst function in Section 2. Discussions are given in Section 3, which is followed by conclusions.

\section{Multi-fractality of Real Traffic}

A direct generalization of $\mathrm{fBm}$ to multifractional Brownian motion $(\mathrm{mBm})$ can be carried out by replacing the Hurst index with a function $H(t)$, satisfying $H:[0, \infty] \rightarrow(0,1)$. This was first carried out independently by Peltier and Levy-Vehel [27] and Benassi, Jaffard and Roux [31] based on the moving average and harmonizable definitions respectively. Following [24] and [26], we define $\mathrm{mBm} X(t)$ by Eq. (1), where $t>0$ and $H:[0, \infty] \rightarrow(a, b) \subset(0,1)$ is a Holder function of exponent $\beta>0$, and $B(t)$ is the standard Brownian motion. The variance of $B_{H(t)}$ is given by Eq. (2), where $\sigma_{H(t)}^{2}=\frac{\Gamma(2-H(t)) \cos (\pi H(t))}{\pi H(t)(2 H(t)-1)}$. Since $\sigma$ is time-dependent, 
it will be desirable to normalize $B_{H(t)}$ such that $E\left[(X(t))^{2}\right]=|t|^{2 H(t)}$ by replacing $X(t)$ with $X(t) / \sigma_{H(t)}$.

$$
\begin{gathered}
X(t)=\frac{1}{\Gamma(H(t)+1 / 2)} \int_{-\infty}^{0}\left[(t-s)^{H(t)-1 / 2}-(-s)^{H(t)-1 / 2}\right] d B(s) \\
+\int_{0}^{t}(t-s)^{H(t)-1 / 2} d B(s) . \\
E\left[(X(t))^{2}\right]=\sigma_{H(t)}^{2}|t|^{2 H(t)} .
\end{gathered}
$$

For the subsequent discussion, $X(t)$ will be used to denote the normalized process. The explicit expression of the covariance of $X(t)$ can be calculated by

$$
\begin{gathered}
E\left[X\left(t_{1}\right) X\left(t_{2}\right)\right]= \\
N\left(H\left(t_{1}\right), H\left(t_{2}\right)\right)\left[\left|t_{1}\right|^{H\left(t_{1}\right)+H\left(t_{2}\right)}+\left|t_{2}\right|^{H\left(t_{1}\right)+H\left(t_{2}\right)}-\left|t_{1}-t_{2}\right|^{H\left(t_{1}\right)+H\left(t_{2}\right)}\right],
\end{gathered}
$$

where

$$
N\left(H\left(t_{1}\right), H\left(t_{2}\right)\right)=\frac{\Gamma\left(2-H\left(t_{1}\right)-H\left(t_{2}\right)\right) \cos \left(\pi \frac{H\left(t_{1}\right)+H\left(t_{2}\right)}{2}\right)}{\pi\left(\frac{H\left(t_{1}\right)+H\left(t_{2}\right)}{2}\right)\left(H\left(t_{1}\right)+H\left(t_{2}\right)-1\right)} .
$$

With the assumption that $H(t)$ is $\beta$-Holder function such that $0<\inf (H(t)) \leq$ $\sup (H(t))<(1, \beta)$, one may approximate $H(t+\rho u) \approx H(t)$ as $\rho \rightarrow 0$. Therefore, the local covariance function of the normalized $\mathrm{mBm}$ has the following limiting form

$$
E[X(t+\tau) X(t)] \sim \frac{1}{2}\left(|t+\tau|^{2 H(t)}+|t|^{2 H(t)}-|\tau|^{2 H(t)}\right), \quad \tau \rightarrow 0
$$

The variance of the increment process becomes

$$
E\left\{[X(t+\tau)-X(t)]^{2}\right\} \sim|\tau|^{2 H(t)}, \tau \rightarrow 0
$$

implying that the increment processes of $\mathrm{mBm}$ is locally stationary. It follows that the local Hausdorff dimension of the graphs of $\mathrm{mBm}$ is given by

$$
\operatorname{dim}\{X(t), t \in[a, b]\}=2-\min \{H(t), t \in[a, b]\}
$$

for each interval $[a, b] \subset R^{+}$.

Due to the fact that the Hurst index $H$ is time-dependent, $\mathrm{mBm}$ fails to satisfy the global self-similarity property and the increment process of $\mathrm{mBm}$ does not satisfy the 
stationary property. Instead, standard $\mathrm{mBm}$ now satisfies the local self-similarity. Recall that $\mathrm{fBm} B_{H}(t)$ is a self-similar Gaussian process with $B_{H}(a t)$ and $a^{H} B_{H}(t)$ having identical finite-dimensional distributions for all $a>0$. For a locally self-similar process, therefore, one may hope that the following expression can provide a description for the local self-similarity of $X(t)$ :

$$
X(a t) \cong a^{H(t)} X(t), \forall a>0,
$$

where $\cong$ stands for equality in distribution. However, this definition of locally self-similar property would lead to a situation where the law of $X(s)$ depends on $H(t)$ when $s$ is far away from $t: X(s) \cong(s / t)^{H(t)} X(t)$. A more satisfactory way of characterizing this property is the locally asymptotical self-similarity introduced by Benassi, Jaffard and Roux [31]. A process $X(t)$ indexed by the Holder exponent $H(t) \in C^{\beta}$ such that $H(t):[0, \infty] \rightarrow(0,1)$ for $t \in R$ and $\beta>\sup (H(t))$ is said to be locally asymptotically self-similar (lass) at point $t_{0}$ if

$$
\lim _{\rho \rightarrow 0_{+}}\left(\frac{X\left(t_{0}+\rho u\right)-X\left(t_{0}\right)}{\rho^{H\left(t_{0}\right)}}\right)_{u \in R} \cong\left(B_{H\left(t_{0}\right)}(u)\right)_{u \in R},
$$

where the equality in law is up to a multiplicative deterministic function of time and $B_{H\left(t_{0}\right)}$ is the $\mathrm{fBm}$ indexed by $H\left(t_{0}\right)$. It can be shown that $\mathrm{mBm}$ satisfies such a locally self-similar property. In passing, the property described by (8) is also analyzed in our recent work [32] from a view of the Cauchy class.

Based on the local growth of the increment process, one may write a sequence

$$
S_{k}(j)=\frac{m}{N-1} \sum_{j=0}^{j+k}|X(i+1)-X(i)|, \quad 1<k<N,
$$

where $m$ is the largest integer not exceeding $N / k$. The local Hurst function $H(t)$ at point $t=j /(N-1)$ is then given by

$$
H(t)=-\frac{\log \left(\sqrt{\pi / 2} S_{k}(j)\right)}{\log (N-1)} .
$$

The function $H(t)$ in (10) can serve as a numerical model of multi-fractality of traffic. Now, we select 4 widely-used real-traffic traces in computer networks. They are DEC-PKT-n.tcp $(\mathrm{n}=1,2,3,4)$ [33]. Fig. $\mathrm{n}(\mathrm{a})$ shows their time series, where $X(i)$ implies the number of bytes in the ith packet $(i=0,1,2, \ldots)$. Fig. $\mathrm{n}$ (b) illustrates the corresponding local Hurst function.

Recall that the $\mathrm{mBm}$ is a locally self-similar process. For $H(t)$ which is a continuous deterministic function, the resulting $\mathrm{mBm}$ is a multi-scale process. On the other hand, if $H(t)$ is a random function or a random process, then the $\mathrm{mBm}$ is a 


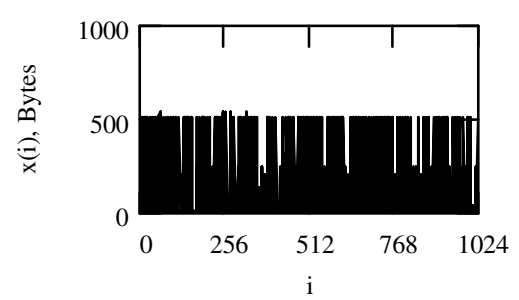

(a)

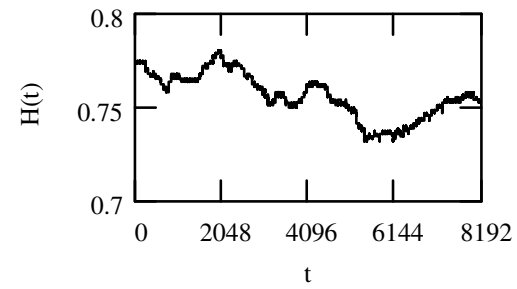

(b)

Fig. 1. (a) Traffic time serie $s X(i)$ of DEC-PKT-1.tcp. (b) Local Hurst function of $X(i)$

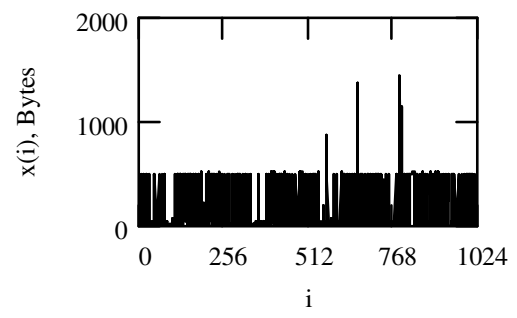

(a)

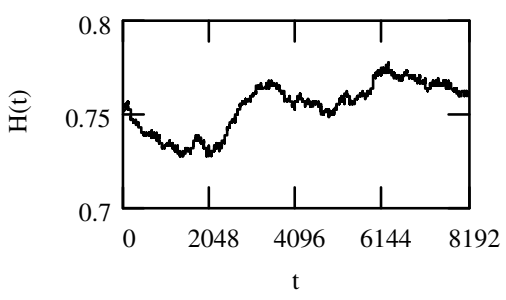

(b)

Fig. 2. (a) Traffic time series $X(i)$ of DEC-PKT-2.tcp. (b) Local Hurst function of $X(i)$

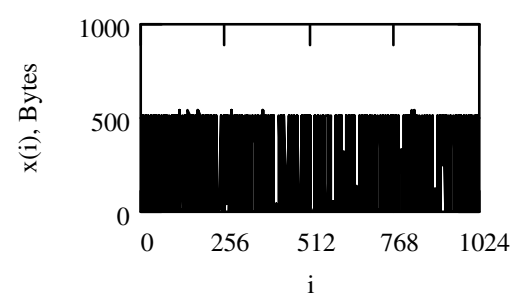

(a)

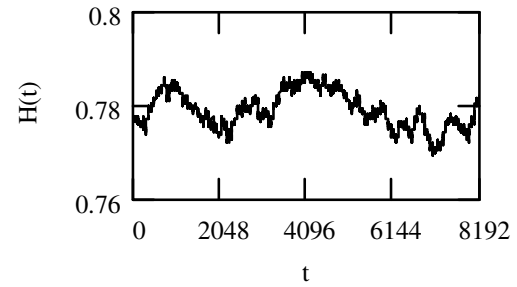

(b)

Fig. 3. (a) Traffic time series $X(i)$ of DEC-PKT-3.tcp. (b) Local Hurst function of $X(i)$

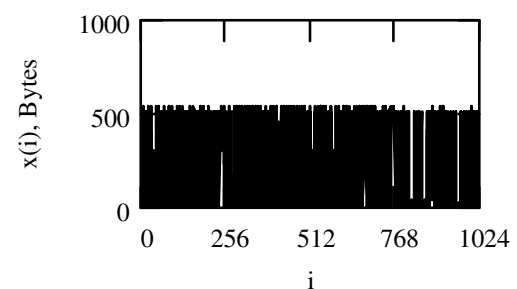

(a)

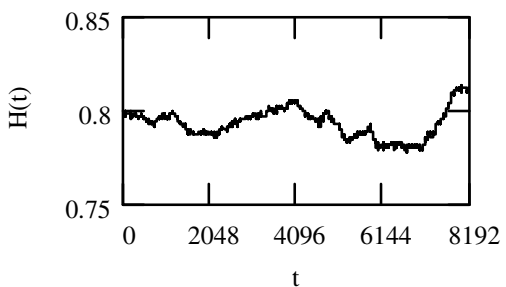

(b)

Fig. 4. (a) Traffic time series $X(i)$ of DEC-PKT-4.tcp. (b) Local Hurst function of $X(i)$ 
Multifractal process. From the above figures, it is obviously seen that $H(t)$ appears random. Thus, $H(t)$ s illustrated in Fig. 1 (b) $\sim$ Fig. 4 (b) are numerical models of multi-fractality of real traffic DEC-PKT-n.tcp $(n=1,2,3,4)$.

\section{Discussions}

The previous figures provide verifications that traffic has the nature of multi-fractal. Recall that the Hurst parameter characterizes the burstness of process from a view of networking. From the above figures, we see that the local Hurst function of a real-traffic series is time varying. Thus, a traffic series may have different burstness at different points of time. As known, if the space of a buffer is occupied, some packets will be dropped and they will be retransmitted late when traffic load becomes light but these packets suffer from delay. The above $H(t)$ s provide evidences that, during a communication process, why packets are delayed due to traffic congestion and why delay is usually a random variable.

Further, the local Hurst functions in Figs. 1 (b) 4 (b) verify that traffic has strictly alternating $O N / O F F$-period behavior, where the term "strictly alternating $O N$ - and $O F F$ - periods" implies 1) the length of $O N$-periods are independent and identically distributed (i.i.d.) and so are the length of $O F F$-periods, and 2) an $O F F$-period always follows an $O N$-period [16].

The assumption that the Hurst function is continuous implies $H(t+\tau) \approx H(t)$ for $\tau \rightarrow 0$. Therefore, the normalized $\mathrm{mBm}$ has the covariance in the following limiting form:

$$
E[X(t+\tau) X(t)]=0.5\left(|t|^{2 H(t)}+|t+\tau|^{2 H(t)}-|\tau|^{2 H(t)}\right), \quad \tau \rightarrow 0
$$

The variance of the increment process is given by

$$
E\left[|X(t+\tau) X(t)|^{2}\right]=|\tau|^{2 H(t)}, \tau \rightarrow 0
$$

Therefore, one sees that the increment process of $\mathrm{mBm}$ is locally stationary.

In practice, a process is causal. Hence, we consider $X_{+}(t)$ which stands for a causal process with the starting point $t=0$. In this case, one has the one-sided $\mathrm{mBm}$ based on fractional integral of Riemann-Liouville type as follows [30]:

$$
X_{+}(t)=\frac{1}{\Gamma(H(t)+1 / 2)} \int_{0}^{t}(t-u)^{H(t)-0.5} d B(u) .
$$

For $t_{1}<t_{2}$, we have the covariance

$$
\begin{gathered}
E\left[X_{+}\left(t_{1}\right) X_{+}\left(t_{2}\right)\right]= \\
\frac{2 t_{1}^{H\left(t_{1}\right)+0.5} t_{2}^{H\left(t_{2}\right)-0.5}}{\left(2 H\left(t_{1}\right)+1\right) \Gamma\left(H\left(t_{1}\right)+0.5\right) \Gamma\left(H\left(t_{2}\right)+0.5\right)} \times{ }_{2} F_{1}\left(0.5-H\left(t_{2}\right), 1, H\left(t_{1}\right)+1.5, t_{1} / t_{2}\right) .
\end{gathered}
$$


The variance of $X_{+}(t)$ has the similar form as that of $X(t)$, i.e., $\sim|t|^{2 H(t)}$ up to a deterministic (or random) function of $H(t)$.

Though the previous discussed $H(t)$ appears time dependent as can be seen from Figs. 1-4, its analytic model remains unknown. Clearly, the multi-fractality of traffic may be quantitatively modelled if analytic models, either deterministic or statistic, of $H(t)$ achieve. Either is greatly desired in practical applications such as pattern recognition of traffic as can be seen from [12], [19]. Finding analytic models of $H(t)$ is our further aim that is certainly attractive.

\section{Conclusions}

We have given and demonstrated the multi-fractality of real traffic based on the local Hurst functions. The present results exhibit that the local Hurst functions of investigated real traffic show that $H(t)$ of traffic is time-varying. The significance of the present results is not only to show multifractal phenomena of traffic on a point-by-point basis instead of an interval-by-interval basis as conventionally done in computer networks but, more importantly, to make the research of the multi-fractality of traffic a step further towards modeling multi-fractality of traffic.

\section{Acknowledgements}

This work was supported in part by the National Natural Science Foundation of China under the project grant numbers 60573125 and 60672114, by the Key Laboratory of Security and Secrecy of Information, Beijing Electronic Science and Technology Institute under the project number KYKF 200606 of the open founding. SC Lim would like to thank the Malaysia Ministry of Science, Technology and Innovation for the IRPA Grant 09-99-01-0095 EA093, and Academy of Sciences of Malaysia for the Scientific Advancement Fund Allocation (SAGA) P 96c.

\section{References}

1. Tobagi, F.A.; Gerla, M., Peebles, R.W., Manning, E.G.: Modeling and Measurement Techniques in Packet Communication Networks. Proc. the IEEE 66 (1978) 1423-1447

2. Jain, R., Routhier, S.: Packet Trains-Measurements and a New Model for Computer Network Traffic. IEEE Journal on Selected Areas in Communications 4 (1986) 986-995

3. Csabai, I.: 1/f Noise in Computer Network Traffic. J. Phys. A: Math. Gen. 27 (1994) L417-L421

4. Paxson V., Floyd, S.: Wide Area Traffic: The Failure of Poison Modeling. IEEE/ACM T. Networking 3 (1995) 226-244

5. Beran, J., Shernan, R., Taqqu, M. S., Willinger, W.: Long-Range Dependence in Variable Bit-Rate Video Traffic. IEEE T. Communications 43 (1995) 1566-1579

6. Crovella, E., Bestavros, A.: Self-Similarity in World Wide Web Traffic: Evidence and Possible Causes. IEEE/ACM T. Networking 5 (1997) 835-846 
7. Tsybakov, B., Georganas, N. D.: Self-Similar Processes in Communications Networks. IEEE T. Information Theory 44 (1998) 1713-1725

8. Li, M., Jia, W., Zhao, W.: Correlation Form of Timestamp Increment Sequences of Self-Similar Traffic on Ethernet. Electronics Letters 36 (2000) 1168-1169

9. Li, M., Jia, W., Zhao, W.: Simulation of Long-Range Dependent Traffic and a TCP Traffic Simulator. Journal of Interconnection Networks 2 (2001) 305-315

10. Li, M., Chi, C.-H.: A Correlation-Based Computational Method for Simulating Long-Range Dependent Data. J. Franklin Institute 340 (2003) 503-514

11. Li, M., Zhao, W., Jia, W., Long, D.-Y., Chi, C.-H.: Modeling Autocorrelation Functions of Self-Similar Teletraffic in Communication Networks Based on Optimal Approximation in Hilbert Space. Applied Mathematical Modelling 27 (2003) 155-168

12. Li, M.: An Approach to Reliably Identifying Signs of DDOS Flood Attacks Based on LRD Traffic Pattern Recognition. Computer \& Security 23 (2004) 549-558

13. Cappe, O., Moulines, E., Pesquet, J.-C., Petropulu, A., Yang X.: Long-Range Dependence and Heavy Tail Modeling for Teletraffic Data. IEEE Sig. Proc. Magazine 19 (2002) 14-27

14. Feldmann, A., Gilbert, A. C., Willinger, W., Kurtz, T. G.: The Changing Nature of Network Traffic: Scaling Phenomena. Computer Communications Review 28 (1998) 5-29

15. Willinger, W., Paxson, V.: Where Mathematics Meets the Internet. Notices of the American Mathematical Society 45 (1998) 961-970

16. Willinger, W., Paxson, V., Riedi, R. H., Taqqu, M. S.: Long-Range Dependence and Data Network Traffic, Long-Range Dependence: Theory and Applications. P. Doukhan, G. Oppenheim, and M. S. Taqqu, eds., Birkhauser (2002)

17. Abry, P., Baraniuk, R., Flandrin, P., Riedi, R., Veitch, D.:, Multiscale Nature of Network Traffic. IEEE Signal Processing Magazine 19 (2002) 28-46

18. Nogueira, A., Salvador, P., Valadas, R.: Telecommunication Systems 24 (2003) 339-362

19. Li, M.: Change Trend of Averaged Hurst Parameter of Traffic under DDOS Flood Attacks. Computers \& Security 25 (2006) 213-220

20. Tsybakov, B., Georganas, N. D.: On Self-Similar Traffic in ATM Queues: Definitions, Overflow Probability Bound, and Cell Delay Distribution. IEEE/ACM T. Networking 5 (1997) 397-409

21. Kim, S., Nam, S. Y., Sung, D. K.: Effective Bandwidth for a Single Server Queueing System with Fractional Brownian Input. Performance Evaluation 61 (2005) 203-223

22. Li, M., Lim, S. C.: Modeling Network Traffic Using Cauchy Correlation Model with Long-Range Dependence. Modern Physics Letters B 19 (2005) 829-840

23. Li, M.: Modeling Autocorrelation Functions of Long-Range Dependent Teletraffic Series Based on Optimal Approximation in Hilbert Space-a Further Study. Applied Mathematical Modelling 31 (2007) 625-631

24. Mandelbrot, B. B.: Gaussian Self-Affinity and Fractals. Springer (2001)

25. Levy-Vehel, J., Lutton, E., Tricot C. (Eds).: Fractals in Engineering. Springer (1997)

26. Peltier, R. F., Levy-Vehel, J.: A New Method for Estimating the Parameter of Fractional Brownian Motion. INRIA TR 2696 (1994)

27. Peltier, R. F., Levy-Vehel, J.: Multifractional Brownian Motion: Definition and Preliminaries Results. INRIA TR 2645 (1995)

28. Muniandy, S. V., Lim, S. C.: On Some Possible Generalizations of Fractional Brownian Motion. Physics Letters A226 (2000) 140-145

29. Muniandy, S. V., Lim, S. C., Murugan, R.: Inhomogeneous Scaling Behaviors in Malaysia Foreign Currency Exchange Rates. Physica A301 (2001) 407-428 
30. Muniandy, S. V., Lim, S. C.: Modelling of Locally Self-Similar Processes Using Multifractional Brownian Motion of Riemann-Liouville Type. Phys. Rev. E 63 (2001) 046104

31. Benassi, A., Jaffard, S., Roux, D.: Elliptic Gaussian Random Processes. Revista Mathematica Iberoamericana 13 (1997) 19-90

32. Lim S. C., Li, M.: Generalized Cauchy Process and Its Application to Relaxation Phenomena. Journal of Physics A: Mathematical and General 39 (2006) 2935-2951

33. http://www.acm.org/sigcomm/ITA/

34. Floyd, S., Paxson, V.: Difficulties in Simulating the Internet. IEEE/ACM T. Networking 9 (2001) 392-403

35. Willinger, W., Govindan, R., Jamin, S., Paxson V., Shenker, S.: Scaling Phenomena in the Internet: Critically Examining Criticality. Proc. Natl. Acad. Sci. USA 99 (Suppl. 1) (2002) 2573-2580 